Mann Whitney $U$ test. A total PsAID score below 4 out of 10 is considered a 'patient-acceptable state'.

Results: A total 95 patients (female $n=53,56 \%$ ) completed the PSAID; TNFi $n=72$ (female $50 \%$, adalimumab $n=41$, Etanercept $n=24$, Golimumab $n=4$, Infliximab $n=2$, Certolizumab $n=1$ ) and non-TNFi $n=23$ (female $74 \%$, Secukinumab $\mathrm{n}=9$, Ixekizumab $\mathrm{n}=1$, Ustekinumab $\mathrm{n}=9$, Tofacitinib $\mathrm{n}=4$ ). The mean age was 53.6 (TNFi 53.5, non-TNFi 53.7) years, and duration of time on bDMARD treatment was TNFi 49.5 (range 1- 141) months, non-TNFi 25.3 (range 4 -59) months. The total and individual domain PsAID scores are shown in the Table 1. A patient acceptable state' total score $<4$ was recorded in TNFi 36/72 (50\%) and non-TNFi $11 / 23(48 \%)$. There was no significant difference between TNFi and non-TNFi groups in the mean total PsAID score, or proportion achieving a patient acceptable state. Patients on TNFi had lower (better outcome) mean scores for all 12 domains except skin, and the differences, versus non-TNFi treated patients, were significant for pain, functional capacity, discomfort and depression.

\begin{tabular}{llll}
\hline PSAID domain & TNFi & Non-TNFi & P value \\
\hline Total score & 3.31 & 4.64 & N.S. \\
Pain & 3.67 & 5.43 & 0.02 \\
Fatigue & 4.04 & 5.65 & N.S. \\
Skin problems & 3.11 & 2.78 & N.S. \\
Work/leisure activities & 3.56 & 4.78 & N.S. \\
Functional capacity & 3.29 & 5.04 & 0.02 \\
Discomfort & 3.88 & 5.65 & 0.02 \\
Sleep disturbance & 3.42 & 4.78 & N.S. \\
Coping & 3.01 & 4.09 & N.S. \\
Anxiety, fear, uncertainty & 2.64 & 4.17 & N.S. \\
Embarrassment/shame & 2.28 & 3.39 & N.S. \\
Social participation & 2.6 & 3.48 & N.S. \\
Depression & 2.11 & 3.91 & 0.03 \\
\hline
\end{tabular}

Conclusion: In PsA, TNFi appear to have a greater impact over non-TNFi bDMARDs on some aspects of quality of life, including pain and functional capacity. TNFi and non-TNFi were no different with respect to patients' perspective on skin disease, embarrassment or shame, despite less good cutaneous responses in clinical trials from TNFi agents. Overall, the PSAID tool reveals an unmet burden on quality of life in PsA patients treated with all classes of bDMARDs and JAKi, as $50 \%$ fail to achieve a 'patient acceptable state'. This should prompt scrutiny of the high scoring domains and utilization of additional treatment modalities to achieve better holistic outcomes for PsA patients in routine care.

REFERENCES

[1] PsAID tool: http://pitie-salpetriere.aphp.fr/psaid/raid_psaid_quest_home.php Disclosure of Interests: Atif Rauf: None declared, Catherine Hughes: None declared, Diane Hill: None declared, Patrick Kiely Speakers bureau: Abbvie. DOI: 10.1136/annrheumdis-2021-eular.2887

\section{POS1057 DISCONNECT BETWEEN TREATMENT SWITCHES AND DISEASE ACTIVITY STATE IN EARLY PSORIATIC ARTHRITIS}

J. Luime ${ }^{1}$, M. Kok ${ }^{2}$, I. Tchetverikov ${ }^{3}$, M. Vis ${ }^{1}$, C. Collaboration of Rheumatologists ${ }^{4}$ on behalf of DEPAR studygroup. ${ }^{1}$ ErasmusMC, Rheumatology, Rotterdam, Netherlands: ${ }^{2}$ Maasstad hospital, Rheumatology, Rotterdam, Netherlands; ${ }^{3}$ Albert Schweitzer Hospital, Rheumatology, Dordrecht, Netherlands; ${ }^{4}$ Cicero, Rheumatology, Rotterdam, Netherlands

Background: Receiving adequate treatment early in the disease course is one of the most important prognostic factors to achieve optimal disease outcome in most rheumatological conditions. Treat-to-target strategies and early step-up of treatment are important ingredients to accomplish this. While for Rheumatoid Arthritis this is common practice on accepted targets, for Psoriatic Arthritis (PsA) this is less outspoken practice. In PsA multiple domains must be taken into account and also there is a less established practice to use PsA specific targets, such as MDA, although this is associated with a better quality of life.[1,2] Whether this affects optimal disease outcome is not well described

Objectives: To describe treatment strategies in newly diagnosed PsA with at least one synovitis and, treatment switches over time in those that not achieved MDA.

Methods: For this study we used data from the DEPAR study between August 2013 and February 2019. The DEPAR study is a cohort that includes newly diagnosed PsA patients and follows them for up to 6 years. Patients are classified at baseline according to their prominent features: mono-, oligo-, polyarthritis, enthesitis, dactylitis or axial phenotype. For this study we used the data from the first 2 years for the patients with an articular phenotype. Methotrexate (MTX) and MTX plus another s-DMARD or GCs were addressed separately as they form the cornerstone of treatment in PSA. Next treatment was grouped in the usual drug classes: sDMARD (conventional), bDMARD and Glucocorticoids (GCs). Descriptive statistics were applied in STATA16 and Tableau 2020.3

Results: Out of 620 patients, 478 patients had at least one swollen joint at baseline and were included in this analysis. 349 (73\%) had data at 1 year and 267
(56\%) at 2 years. Average age was 50.3 years(sd 14.1 ), and $50 \%$ was female. Half of the patients had oligo-arthritis, $30 \%$ poly-arthritis and $20 \%$ mono-arthritis. At baseline, clinical characteristics were 3.8 swollen joints (sd 4.9), 5.5 tender joints (sd 6.2), 3.1 PASI points (sd 3.7, skin), 0.8 entheseal points (LEl; sd 1.2) and 0.1 dacylitis count (basic count of digits; sd 0.6). Treatment was initiated with Monotherapy MTX in $51 \%$ of the patients, while $25 \%$ did not start any DMARD, $10 \%$ started a combination of s-DMARDs, $9 \%$ a single s-DMARD, $3 \%$ a b-DMARD and $2 \%$ GCs. This differed for the different phenotypes as shown in Table 1.

Minimal Disease Activity (MDA) was achieved by $32 \%$ after 3 months increasing to $58 \%$ at 24 months. Treatment switches in the non-MDA mono-, oligo- and polyarthritis groups are shown in Figure 1. In the patients not in MDA treatment was not switch in: $70 \%$ of patients with mono-arthritis, $50-66 \%$ with oligo-arthritis and $50 \%$ poly-arthritis. Similar observations were found when using the DAS28 $<=3.2)$ or DAPSA $(<14)$, both including only articular disease activity (data not shown). Conclusion: Achieving MDA is associated with a better quality of life, though it is not yet common practice to make treatment adjustments based on MDA. Of the newly diagnosed PsA patients not in MDA, more than half of the clinical evaluations did not result in a switch of medication.

REFERENCES:

[1] Wervers, K, et al ART 2019

[2] Coates, L, et al. Lancet 2015

Monoarthritis not in MDA

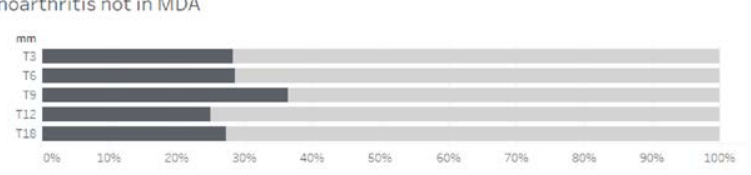

Oligoarthritis not in MDA

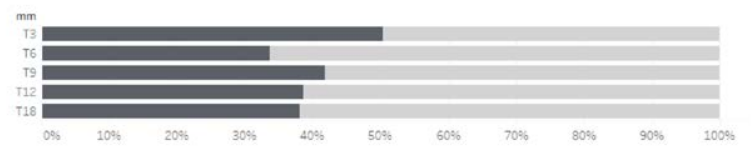

Polyarthritis - not in MDA

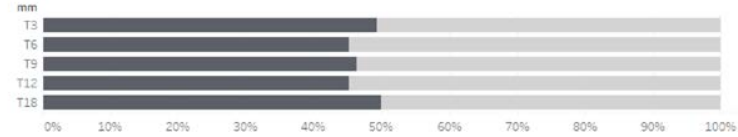

Figure 1. Medication change in patients not in MDA at each timepoint (dark bars = change and light bars $=$ no change)

table 1 baseline medication

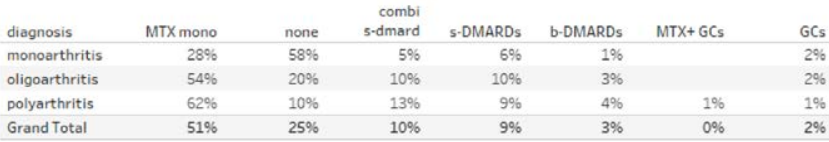

Disclosure of Interests: Jolanda Luime: None declared, Marc Kok Grant/ research support from: Pfizer, Novartis, BMS, Abbvie, Ilja Tchetverikov: None declared, Marijn Vis Grant/research support from: Novartis, CICERO collaboration of rheumatologists: None declared.

DOI: 10.1136/annrheumdis-2021-eular.3748

\section{POS1058 SUBCLINICAL DACTYLITIS: COMPARATIVE STUDY OF THERAPEUTIC RESPONSE WITH TNF INHIBITORS, SECUKINUMAB AND USTEKINUMAB}

\section{Guillén-Astete ${ }^{1}$, Á. Andreu-Suárez ${ }^{1}$, V. García García ${ }^{1}{ }^{1}$ Hospital Ramón y} Cajal, Rheumatology, Madrid, Spain

Background: Non tender dactylitis has been recently associated with synovitis, tenosynovitis and enthesitis without fat tissue hyperemia. Dactylitis is a remark able hallmark of spondyloarthritis and particularly in psoriatic arthritis. Also, dactylitis has been recognized as one of the most difficult musculoskeletal feature of the disease to treat. It has been of interest to compare the effectiveness of available therapies in cases of clinical dactylitis, however, the efficacy on subclinical dactylitis has not studied yet.

Objectives: To determine the effectiveness of three different biological therapy approaches in subclinical (non-tender) dactylitis in patients diagnosed by psoriatic arthritis in terms of changes detected by ultrasound scan. 
Methods: A retrospective ultrasound record-based study was conducted to compare the findings of ultrasonographic studies performed before and 6 months after the start of one of the following biologic therapies in patients diagnosed by psoriatic arthritis: TNF-inhibitors, secukinumab and ustekinumab. We included patients with a complete before-after ultrasound scan of the hands from three different rheumatology clinics. To be considered as a subclinical dactylitis two conditions were had to be achieved: non tender finger and absence of fat tissue hyperemia.

Results: Patients included: 22 on TNF-inhibitors (28 subclinical dactylitis), 14 on secukinumab (17 subclinical dactylitis) and 13 on ustekinumab (16 subclinical dactylitis). Median of line of treatment of each biologic therapy: 1, 3 and 3 , respectively. In the TNF-inhibtors group, 14/28 achieved synovitis resolution, $12 / 26$ achieved tenosynovitis resolution and 2/28 achieved enthesitis resolution. In the secukinumab group, 11/17 achieved synovitis resolution, 11/17 achieved tenosynovitis resolution and 13/17 achieved enthesitis resolution. In the ustekinumab group, 10/16 achieved synovitis resolution, 10/15 achieved tenosynovitis resolution and 13/16 achieved enthesitis resolution. Complete dactylitis resolution was achieved in 12/28, 11/17 and 10/16 patients, respectively. Differences on independent success ratio of secukinumab and ustekinumab were not statistically significative, however both groups had higher rates of independent success compared to TNF-inhibitors group ( $\mathrm{P}<0.05$, both cases). The line of treatment did not affect the resolution rate of non-TNF-inhibitors group.

Conclusion: This is -as far as our knowledge know- the first study comparing the effect of different therapies on subclinical dactylitis. Besides the inherit limitations of a retrospective study, it seems to point that both secukinumab and ustekinumab are better therapeutic options than TNF-inhibitors. Of course, prospective further studies are needed.

Disclosure of Interests: None declared.

DOI: 10.1136/annrheumdis-2021-eular.4203

\section{Psoriatic arthritis - clinical aspects (other than treatment)}

\section{POS1059 ULTRASOUND SYNOVITIS, UNLIKE ENTHESITIS OR CLINICAL JOINT ASSESSMENT, IS ASSOCIATED WITH JOINT DAMAGE PROGRESSION IN PATIENTS WITH PSORIATIC ARTHRITIS}

Y. Yamada ${ }^{1}$, K. Inui ${ }^{2}$, T. Okano ${ }^{1}$, K. Mandai ${ }^{2}$, K. Mamoto $^{1}$, T. Koike $^{1}$, C. Tateishi ${ }^{3}$, D. Tsuruta ${ }^{3}$, H. Nakamura ${ }^{1} .{ }^{1}$ Osaka City University Graduate School of Medicine, Department of Orthopedic Surgery, Osaka city, Japan; ${ }^{2}$ Saiseikai Nakatsu Hospital, Department of Orthopedic Surgery, Osaka city, Japan; ${ }^{3}$ Osaka City University Graduate School of Medicine, Department of Dermatology, Osaka city, Japan

Background: We previously reported that ultrasound assessment of enthesitis (US enthesitis) is not consistent with tenderness of the enthesis (clinical enthesitis) in patients with psoriatic arthritis (PsA). Although US enthesitis reflects inflammatory condition and clinical enthesitis is associated with disease activity and physical function, each of them was not associated with joint destruction by cross-sectional analysis1. It is reported that the utility of US for predicting joint destruction remains unclear among patients with PsA2.

Objectives: This study is aimed to longitudinally investigate the relationships between enthesitis or synovitis and joint damage progression in patients with PsA. Methods: Forty-seven patients with PsA (average age of 56.5 years) underwent US and clinical examination of wrist and finger joints and 14 entheses (the bilateral humeral medial epicondyles and insertions of the triceps, distal quadriceps, proximal/distal patella, Achilles tendons, and plantar fascia). Tender or swollen joint count (TJC/SJC), Gray Scale (GS) and Power Doppler (PD) score of the joints, and US/clinical enthesitis counts were calculated. The relationships between the yearly progression in modified total sharp score ( $\triangle \mathrm{mTSS}$ ) at twotime points (baseline and average follow-up of 20.4 months) and US or clinical findings were investigated.

Results: $\triangle \mathrm{mTSS}$ was significantly correlated with age $(\mathrm{r}=0.44, \mathrm{p}=0.01)$, joint $\mathrm{GS}$ score $(r=0.44, p=0.01)$, and joint PD score $(r=0.38, p=0.03)$. TJC, SJC, inflammatory marker, and disease activity showed no associations with $\triangle \mathrm{mTSS}$. US/ clinical enthesitis counts also showed no associations with $\Delta \mathrm{mTSS}$ (Table 1). The joint PD score, adjusted by age, was significant factor for $\triangle \mathrm{mTSS}$ by multiple regression analysis $(b=0.50, p<0.001)$.

Conclusion: The joint PD score or US synovitis, unlike clinical joint assessment, was significant predictive factor for joint damage progression. It is important to assess joints by US as well as clinical examination.

References: 1) Yutaro Yamada et al. Ultrasound assessment, unlike clinical assessment, reflects enthesitis in patients with psoriatic arthritis. Clin Exp Rheumatol. 2020 Apr 17. Online ahead of print.

2) van der Heijde et al. Assessing structural damage progression in psoriatic arthritis and its role as an outcome in research. Arthritis Res Ther. 2020, 22(1): 18.
Table 1. Univariate analysis of predictive factors for joint damage progression in PsA patients.

\begin{tabular}{lllll}
\hline & \multicolumn{2}{l}{ mTSS at baseline } & \multicolumn{2}{l}{$\Delta$ mTSS } \\
& R value & p value & R value & p value \\
\hline age & 0.55 & $<0.001$ & 0.44 & 0.01 \\
PASE & 0.04 & 0.81 & 0.12 & 0.52 \\
PASI & -0.25 & 0.15 & -0.01 & 0.96 \\
DAS28CRP & -0.05 & 0.75 & 0.07 & 0.71 \\
DAPSA & -0.01 & 0.94 & -0.01 & 0.97 \\
HAQ & 0.17 & 0.27 & -0.07 & 0.73 \\
CRP & -0.13 & 0.38 & 0.23 & 0.20 \\
MMP-3 & 0.04 & 0.80 & 0.29 & 0.12 \\
biologics use & 0.19 & 0.19 & -0.11 & 0.54 \\
Clinical enthesitis counts & -0.01 & 0.97 & -0.19 & 0.30 \\
TJC & -0.05 & 0.76 & -0.10 & 0.58 \\
SJC & 0.21 & 0.18 & 0.13 & 0.48 \\
US enthesitis counts & 0.12 & 0.44 & -0.13 & 0.48 \\
joint GS score & 0.08 & 0.60 & 0.44 & 0.01 \\
joint PD score & 0.08 & 0.60 & 0.38 & 0.03 \\
\hline
\end{tabular}

PsA: psoriatic arthritis, mTSS: modified Total Sharp Score, PASE: Psoriatic Arthritis Screening and Evaluation, PASI: Psoriasis Area Severity Index, DAPSA: Disease Activity in Psoriatic Arthritis, DAS: Disease Activity Score, CRP: C-reactive protein, HAQ: Health Assessment Questionnaire, MMP-3: matrix metalloproteinase 3, TJC: tender joint counts, SJC: swollen joint counts, GS: Gray Scale, PD: Power Dopple

Disclosure of Interests: Yutaro Yamada: None declared, Kentaro Inui Speakers bureau: Abbvie, Eisai, Eli Lilly, Grant/research support from: Abbvie, Eisai, Chigai, Eli Lilly, Daiichi Sankyo, Mitusbishi Tanabe, Pfizer, UCB, Tadashi Okano Speakers bureau: Abbvie, Koji Mandai: None declared, Kenji Mamoto: None declared, Tatsuya Koike Grant/research support from: Abbvie, Chugai, Chiharu Tateishi: None declared, Daisuke Tsuruta Speakers bureau: Abbvie, Astellas, Bristol-Myers Squibb, Celgene, Eli Lilly, Janssen, Novartis, Sanofi, Grant/research support from: Abbvie, Eli Lilly, Bristol-Myers Squibb, UCB, Hiroaki Nakamura Grant/ research support from: Astellas, Asahi Kasei DOI: 10.1136/annrheumdis-2021-eular.281

\section{POS1060 A PROSPECTIVE, LONGITUDINAL STUDY TO EVALUATE REAL-WORLD PATIENT EXPERIENCES AND TREATMENT SATISFACTION WITH SECUKINUMAB FOR PSORIATIC ARTHRITIS}

M. E. Husni ${ }^{1}$, M. Bozyczko' ${ }^{2}$, D. Wolin ${ }^{3}$, C. Sweeney ${ }^{3}$, E. Davenport ${ }^{3}$, S. Hass ${ }^{4}$ E. Yi ${ }^{4}$, P. Hur ${ }^{4}$, L. Grinnell-Merrick ${ }^{5}{ }^{1}$ Cleveland Clinic, Rheumatology, Cleveland, United States of America; ${ }^{2}$ National Psoriasis Foundation, Patient Advocate, Portland, United States of America; ${ }^{3}$ RTI Health Solutions, Surveys and Observational Studies, Research Triangle Park, United States of America; ${ }^{4}$ Novartis Pharmaceuticals Corporation, Health Economics and Outcomes Research, East Hanover, United States of America; ${ }^{5}$ University of Rochester, Rheumatology, Rochester, United States of America

Background: Psoriatic arthritis (PsA) is an inflammatory disease that impairs quality of life. Despite therapeutic advances, up to a third of patients do not respond or are unable to sustain a treatment response. Thus, data on patient treatment experience in PsA is critical.

Objectives: To assess real-world experiences of patients with PsA treated with secukinumab.

Methods: This is an ongoing 12-month, prospective, longitudinal, web-based survey study of patients with PsA initiating secukinumab. Patient demographics and medical history are collected at baseline using a rheumatologist case report form. Patient experience data are collected at baseline, 3, 6, 9, and 12 months by patien surveys. The primary endpoint is change in the Psoriatic Arthritis Impact of Disease 12-item questionnaire (PsAID12) from baseline to 3 months. In addition, this study will explore other changes at 3 months, including PsA symptoms, treatment satisfaction with secukinumab, and other patient-reported outcome measures. Baseline and available month 3 data from patients enrolled to date were analyzed descriptively. Results: Baseline demographic and medical history data were available for 72 patients; 32 (44.4\%) were male, $59(81.9 \%)$ were White, and 15 (20.8\%) reported modifying their diet because of PsA. The mean (SD) time since symptom onset $(n=29)$ and since PsA diagnosis $(n=53)$ was $56.3(45.6)$ and $34.4(44.7)$ months, respectively. More than half of the patients who started treatment with secukinumab were biologic experienced (38/62; $61.3 \%)$. Primary and secondary loss of effectiveness were the main reasons for previous biologic discontinuation (Table 1). At 3 months $(n=49)$, the mean (SD) PsAID12 change from baseline was -1.8 (1.8) points. Additionally, $24.5 \%$ of patients reported their PsA symptoms to be "much better," $40.8 \%$ reported "moderately better," and $32.7 \%$ "a little better" compared with baseline; none reported "no change" or worsened symptoms. On a scale of $1-10$, patients rated their overall satisfaction with secukinumab treatment at month 3 with a mean (SD) score of 6.8 (2.1). Patients were also highly 